\title{
Experimental Noise Analysis of Reed Switch Sensor Signal under Environmental Vibration
}

\author{
Odgerel Ayurzana ${ }^{1}$ and Hiesik $\mathrm{Kim}^{2}$ \\ 1. Department of Computer Engineering, Mongolian University of Science and Technology, Ulaanbaatar, Mongolia \\ 2. Department of Electrical and Computer Engineering, University of Seoul, Seoul, Korea
}

\begin{abstract}
The chattering noise problem of reed switch sensor signal for Automatic Meter Reading system was analyzed experimentally under various types of external vibrations and shocks. The external vibration level amplitude was measured with an accelerometer. To apply for water flow measurement devices, the reed switch sensors should keep high reliability. But the measured digital meter data are occurred difference or errors by chattering noise. The reed switch contains chattering error by itself at the force equivalent position. The vibrations such as passing vehicle near to the reed switch installed location causes chattering. In order to reduce chattering error, most system uses just software methods, for example using digital filter algorithm and also statistical calibration methods. However software approaches were implemented for reducing chattering error, there has still generated chattering error due to external mechanical vibrations and magnetic field. The chattering errors can be reduced by changing leaf spring structure using mechanical hysteresis characteristics.
\end{abstract}

Key words: Noise analysis, accelerometer, magnet, vibration, reed switch, sensor, chattering error.

\section{Introduction}

To measure water flow amount for AMR (automatic meter reading) system of water supply, a reed switch, CYBLE, MR (magnetic resistive) and CCD (charge couple device) image sensors are applied in field. The conventional water flow meters of mechanical type have implied many limitations that a person needs to visit the location of the each water meter and write down manually the meter data [1]. Manual reading takes much time, labor and expenses. Each of this method has its own advantages and disadvantages. The MR, CYBLE and image sensor modules are consumed the additional battery. The reed switch sensor runs on without battery that is directly connected to the terminal device. The reed switch sensor has very simple internal and external structure and is easy to apply and mount for any practical fields.

Corresponding author: Odgerel Ayurzana, Ph.D., research fields: sensor networking, embedded systems and applications, signal processing.

Hiesik Kim, Ph.D., research fields: optical measurement of geometries, applications of sensors for automation, image processing.
Also reed switch sensor is much cheaper than other sensor modules. The reed switch sensor contains chattering error seriously by itself internal structure. The purpose of this study is focused on analysis and reduction of chattering error for remote measurement of water supply management. The operation of reed switch sensors can be described as a mechanical contact switch by approximation of permanent magnet piece to generate an electrical pulse. The reed switch sensors are used mostly in measurement application to detect the rotational or translational displacement.

They are applied for the electronic digital type of water flow meters. The reed switch sensor is just mounted simply on the conventional mechanical type flow meter. A small magnet is attached on a pointer of the water meter counter rotor. Inside the reed sensor, two steel leaf springs create mechanical contact and apart repeatedly as rotation of flow meter counter. The counting electrical contact pulses can be converted as the water flow amount. The MCU sends the digital flow rate data to the server using the wireless communication network. But the digital meter data are 
occurred difference or errors by chattering noise.

Two nickel iron blades, sealed hermetically in a glass tube are forming together a contact unit, hereby the magnetically part is equivalent to the electrical part, whenever a magnetic field, constructed by a coil or a permanent magnet is applied to the terminals, magnetic north and south poles are conducted to the contact zone, with the effect that the blades are pulled together. Whenever the magnetic field is weak enough, the blades are released again. The difference between pull in and drop out is named the hysteresis and is determined by the thickness of the non-magnetic surface of the contact zone and of the overlap of these blades. With this behavior the reed contact has a designated snap characteristic. In Ref. [2] paper presents contact materials for reed switches. In Ref. [3] paper discusses the miniaturization limit of the reed switch with respect to the magnetic and mechanical properties. Influence of contact surface conditions of reed switches is investigated in Ref. [4]. To reduce chattering in sliding-mode control, a boundary layer around the switching surface is used, and a continuous control is applied within the boundary [5]. A conventional reed switch is operated by the mechanical force, so there has contained a design limitation. There is generated error by the actual state vibration when magnetic and reed switch are approximated and separated due to force balance condition. Due to the magnetic attached pointer of the water meter counter rotor stops near to the reed switch and also external impacts and vibrations such as passing vehicle near to the reed switch installed location causes additional noise pulses that named as chattering error. In order to remove these additional noise pulses resent systems use just software methods, for example using digital filter algorithm and statistical calibration methods. But there is necessary need to introduce hardware approaches that should upgrade itself hardware structure for reed switch. Some small particles are blocked inside to the gear and wing of the mechanical water flow meter when water flows through the water meter then rotating wing is possible to jam and break down.

\section{Background of Study}

Seoul water works research institute supported the project named as "Introduction strategy for automatic meter reading system for waterworks services" during one year. As a result of this project, the meter data receive rate using reed switch sensor module which was lower than other sensor modules. As shown in the experiment, there was occurred water data difference between remote and human power measurement when implementing the reed switch sensor module for water automatic measurement system.

Water supply companies have developed and implemented their own technology for remote measurement system. As following Fig. 1 shows average meter data receive rates of various kinds of sensor modules which were experimented for remote measurement of water supply. Above mentioned project result of one year, the average meter data receive rate using reed switch sensor module was lower than digital MR and CYBLE sensor modules. The meter data receive rate using the reed switch sensor was decreased due to the chattering error that causes various types of vibrations and magnetic fields [6]. Therefore, there needs a study to analyze and reduce

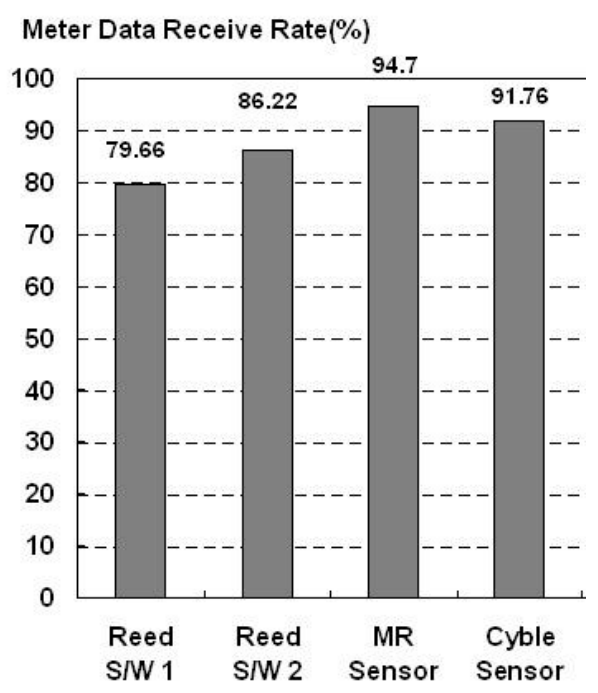

Fig. 1 Comparison of meter data receive rates. 
the chattering error. The chattering means the undesired intermittent when a moment of making and breaking operation of reed springs. It may occur either when the reed contact is operated, released or when subjected to external shock or vibration.

\section{System Design for Chattering Error}

Following a sub-miniature (KSM-1322) and a low-hysteresis (KMC-1825) two types of reed switches were used for chattering experiment.

KSM-1322 is a sub-miniature reed switch. This forms a reed switch which is built with a $2.25 \mathrm{~mm}$ diameter glass having a higher wall thickness for better glass to metal seals, and is a good solution for general purpose, high volume applications with a little shock and vibration. The center contact also makes it suitable for coil applications. The three differential ranges available cover a wide range of releasing specific applications.

KMC-1825 is a low-hysteresis reed switch that is built with specially pressed springs with slightly higher rigidly for close differential, low hysteresis applications where an operate and release are required with minimum magnet travel or minimum change in coil voltage. In order to reduce the hysteresis characteristics, two reed springs of the reed switch are mounted perpendicular each other that means the contact area of the two reed springs less than other types of reed switches.

Fig. 3 shows the main schematic diagram of the vibration experiment system.

An air compressor (SEN 25-1, max pressure-8 $\mathrm{kg} / \mathrm{cm}^{2}$ ) controlled vibrator was designed as a rigid plastic platform. The vibrator system consists of air compressor, air pressure regulator $\left(5 \mathrm{~kg} / \mathrm{cm}^{2}\right)$, pressure meter, solenoid valves, air cylinder tube, left and right velocity regulator valves, left and right limit switches, rail guide and moving table. The air compressor has been provided by high pressure, in our case $5 \mathrm{~kg} / \mathrm{cm}^{2}$ that can be regulated air pressure amount by the pressure regulator (PW 2000). Two connectors of the solenoid valve (TVF 3230) were connected with air cylinder (TCP 1B16-45) by hose (Ø6mm).

The inside of the air cylinder has moving steel shaft
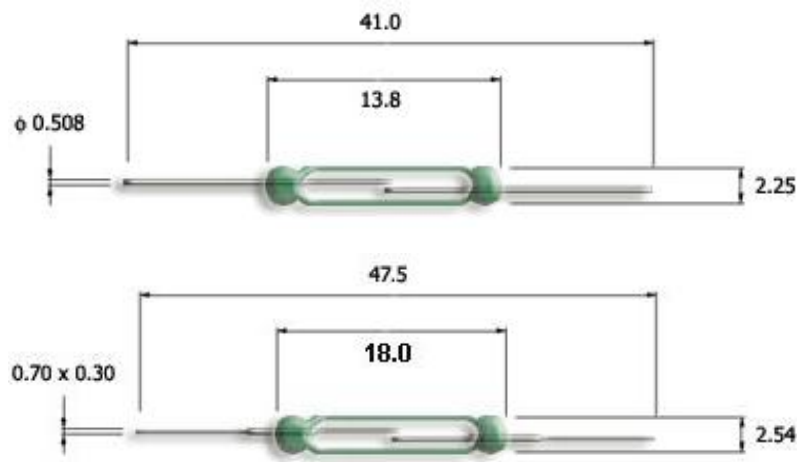

Fig. 2 Internal structure of reed switches (KSM-1322 and KMC-1825).

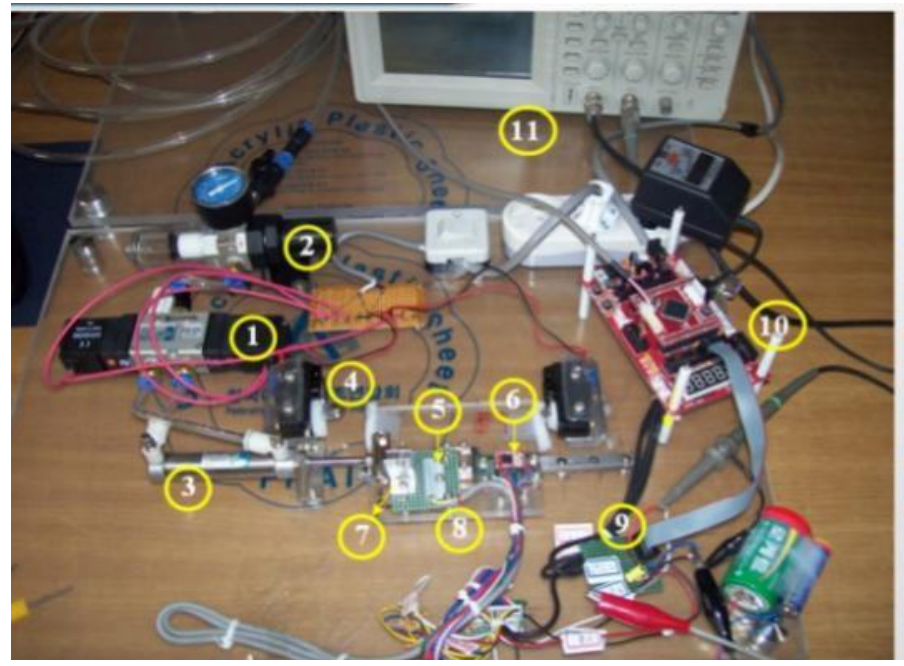

Solenoid valve
Pressure
regulator
Air cylinder
tube
Limit switch
Reed S/W sensor
Acceleration
sensor
Magnetic
Moving table
RC Low Pass
Filter
ATMega128 Kit
Oscilloscope

Fig. 3 Experimental schematic layout of main oscillation table of reed switch vibration experiment. 
(45 mm) for making line motion. This steel shaft of the air cylinder is connected to the moving oscillation table directly for creating the vibration. The vibration velocity has been controlled by left and right regulator valves that are mounted on the air cylinder. That means to regulate high and low air pressure from air compressor. Left and right limit switches are installed at the left and right ends of moving table respectively.

The moving table had a size of $100 \times 80 \times 10 \mathrm{~mm}$ making by acrylic plastic. The acceleration sensor, reed switch sensor and small magnet of ferrite type $(12 \times 12 \times 5 \mathrm{~mm}$, magnetic flux density $\mathrm{B}=633.6$ Gauss $)$ were installed on the moving table for measuring vibration level and chattering error. In order to measure the vibration level, system has utilized a 3 axes acceleration sensor (MMA7260Q). A surface-mounted accelerometer was attached to the plastic acrylic platform to measure horizontal and vertical acceleration of the moving table. The accelerometer is a DC accelerometer having a range of $\pm 6 \mathrm{~g}$. $\mathrm{g}$ is the acceleration of gravity equals to $9.8 \mathrm{~m} / \mathrm{sec}^{2}$.

The ATMega 128 kit was used to convert the reed switch, vertical and horizontal vibration analog signals to the digital signals through the ADC (F port). The input clock frequency of the ADC is $125 \mathrm{KHz}$. The RS232 port rate, data bit, stop bit and parity were set $57,600 \mathrm{bps}, 8$ bit, 1 bit and no parity respectively. As shown in the experiment, 358 sampling numbers of chattering error data were included $30 \mathrm{sec}$. In our case, we have estimated counts of chattering error pulse per every 10 seconds.

After removing noise of the acceleration sensor output, it is necessary to estimate proper vibration amplitude. Vibration amplitude values have to cover all contents and characteristic of the vibration. The RMS approach was used to estimate for quantifying the vibration level. The RMS for a collection of $n$ values $\left\{x_{1}, x_{2}, \ldots . x_{n}\right\}$ is

$$
x_{R M S}=\sqrt{\frac{1}{n} \sum_{i=1}^{n} x_{i}^{2}}=\sqrt{\frac{x_{1}^{2}+x_{2}^{2}+\ldots . .+x_{n}^{2}}{n}}
$$

The corresponding formula for a continuous function $f(t)$ defined over the interval $T_{1} \leq t \leq T_{2}$ is

$$
f_{R M S}=\sqrt{\frac{1}{T_{2}-T_{1}} \int_{T_{1}}^{T_{2}}\left[f(t)^{2}\right] d t}
$$

The RMS of a periodic function is equal to the RMS of one period of the function. The RMS value of a continuous function or signal can be approximated by taking the RMS value of a series of equally spaced samples.

\section{Experiment and Analysis of Chattering Error}

The output signal mixed with much noise when a $\mathrm{RC}$ low pass filter is not connected on the outputs of the acceleration sensor $\mathrm{X}, \mathrm{Y}, \mathrm{Z}$. In order to remove white random noises, RC low pass filter was implemented in the circuit of accelerometer.

According to the input vibration level, there is generated the additional noise pulses named as chattering error.

There were seriously generated the chattering error pulses when the acceleration value increases.

Our experiment device using the air compressor has created much strong vibration levels. As shown in the experiment, maximum and minimum vibration levels were $2.18 \mathrm{~g}$ and $0.11 \mathrm{~g}$ by RMS method respectively. We cannot measure low level of vibration until $0.11 \mathrm{~g}$. Therefore, in order to measure low level of vibration

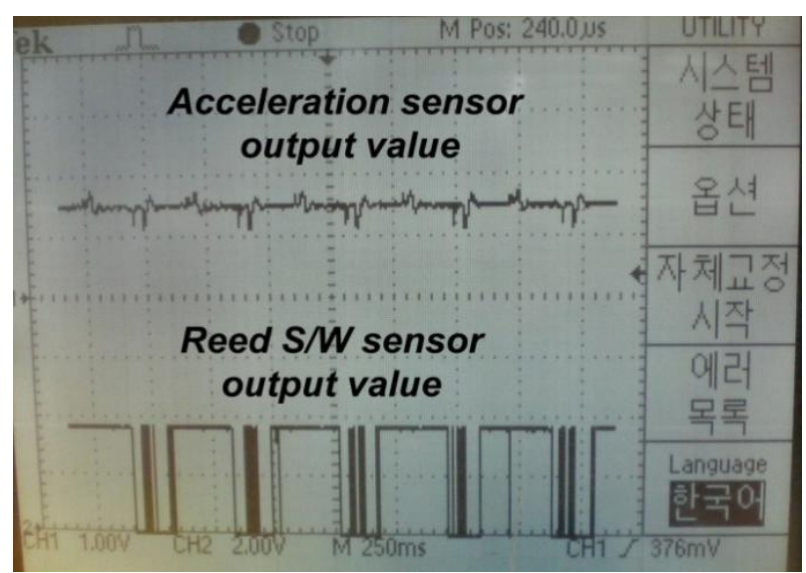

Fig. 4 Low level vibration and chattering error. 


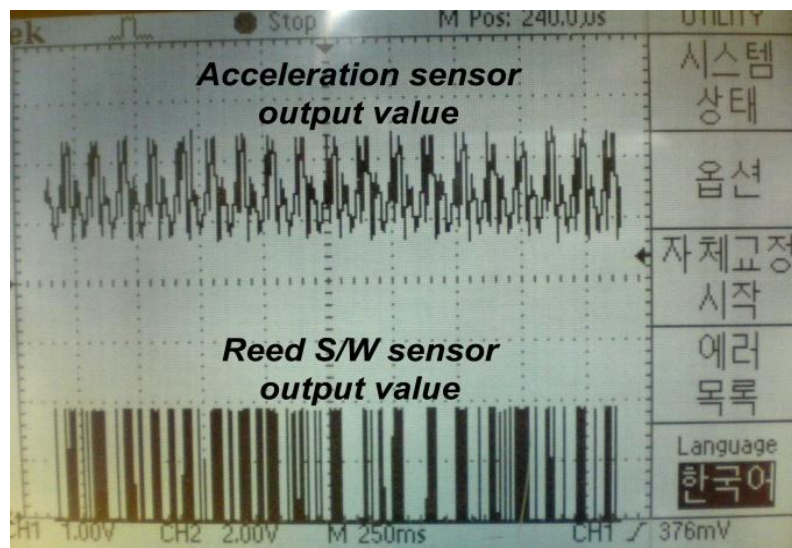

Fig. 5 High level vibration and chattering error.

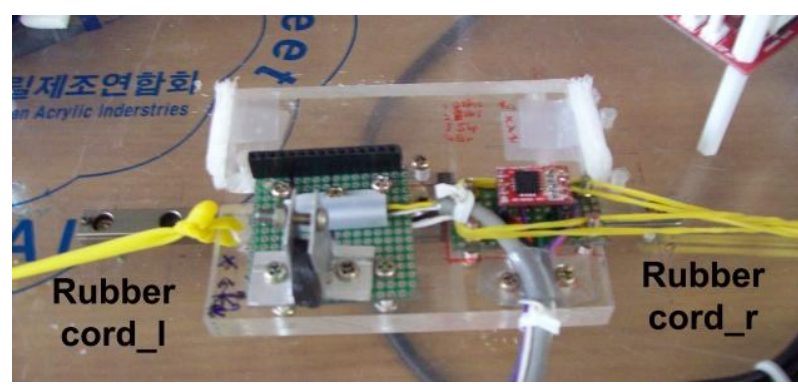

Fig. 6 Chattering experiment table using rubber cords.
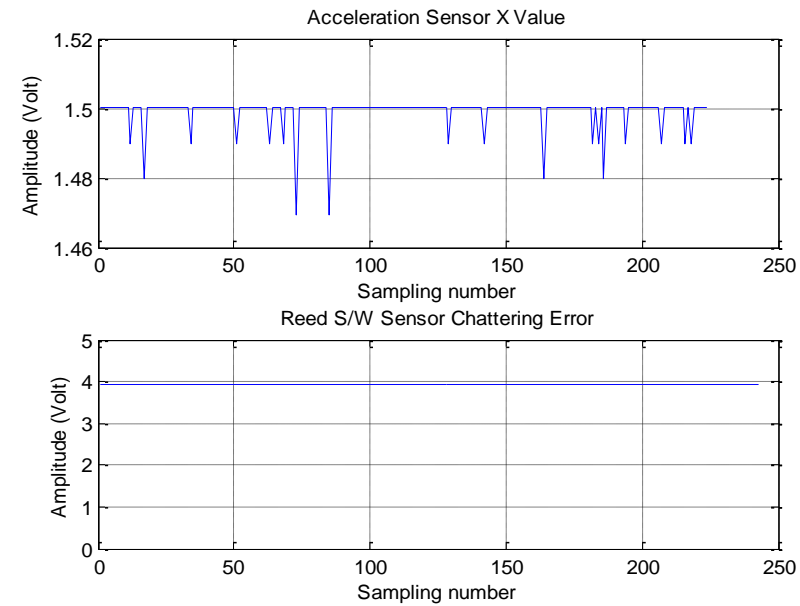

Fig. 7 No chattering error when vibration level was $0.11 \mathrm{~g}$ (KSM-1322).

there is need to design another motion force. The rubber cords were fixed right and left ends of the moving oscillation table for creating low level vibration like following Fig. 6. A person makes vibration with her/his hand mechanically.

In further experiment, a low level of vibration until $0.11 \mathrm{~g}$ was measured using oscillation table as shown in Fig. 6. The vibration table using the air compressor is used from $0.11 \mathrm{~g}$ vibration level value.
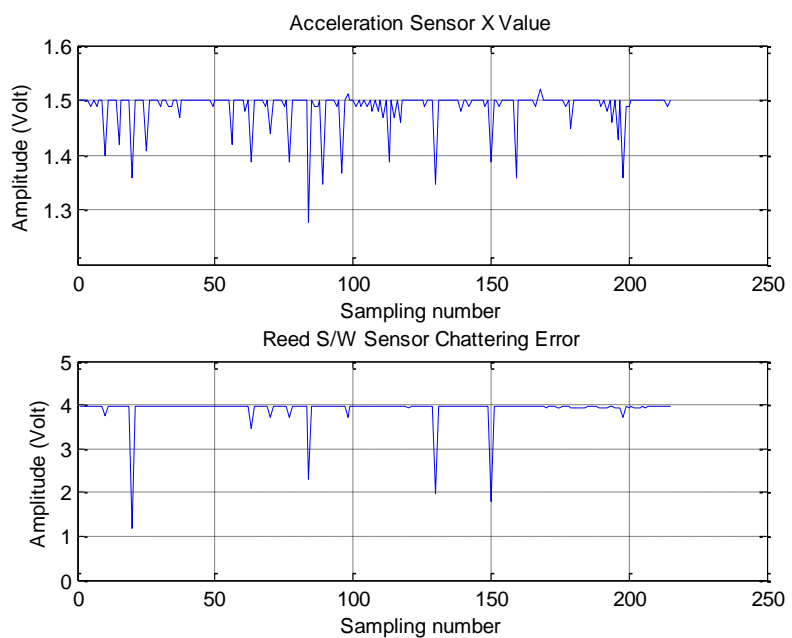

Fig. 8 Generating chattering error when vibration level was $0.15 \mathrm{~g}$ (KSM-1322).

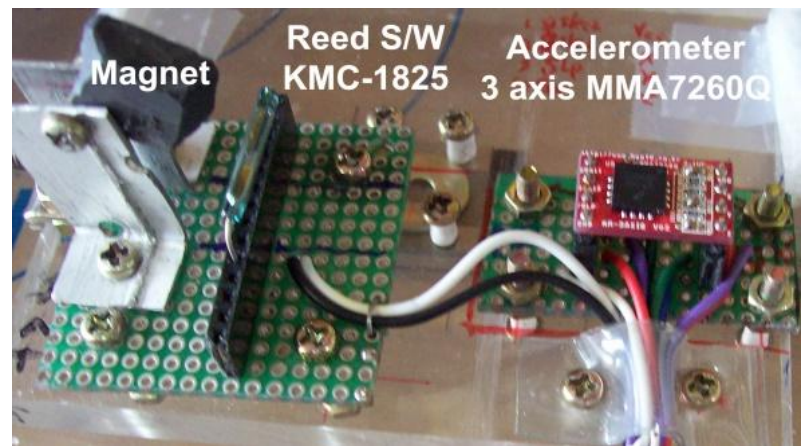

Fig. 9 Layout of a 3-axis accelerometer and a reed switch in $\mathrm{X}$ direction (horizontal vibration).

It's very important to describe beginning vibration level value of chattering error by precisely.

The chattering error pulses were not generated when vibration level value increases until $0.11 \mathrm{~g}$ for KSM-1322.

The chattering error pulses were generated from vibration level value of $0.15 \mathrm{~g}$.

\section{Result for Chattering Error}

The contacting distance between reed switches (KSM-1322, KMC-1825) and ferrite magnets $(\mathrm{B}=$ 633.6 Gauss) was fixed 11 and $7 \mathrm{~mm}$ respectively. This distance of 11 and $7 \mathrm{~mm}$ is a contact moment (On and Off) of the reed springs when the magnet approaches the sensor.

As shown in Fig. 10, vibration bear capability of the low-hysteresis reed switch was better than sub-miniature reed switch. 


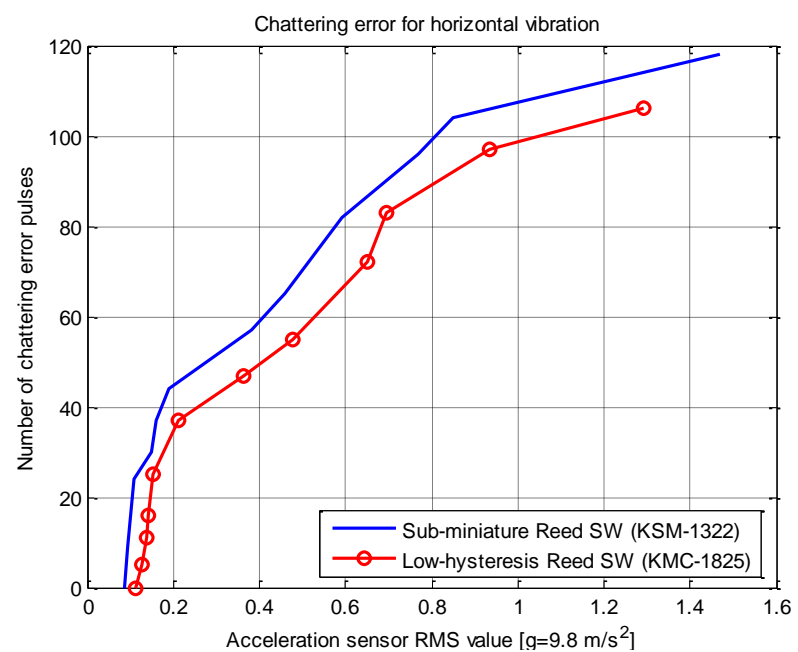

Fig. 10 Chattering error of reed switch for horizontal vibration.

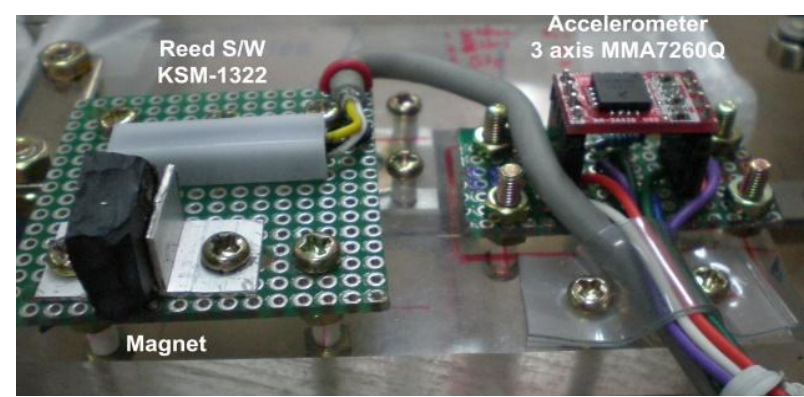

Fig. 11 Layout of a 3-axis accelerometer and a reed switch in $\mathrm{Y}$ direction (vertical vibration).

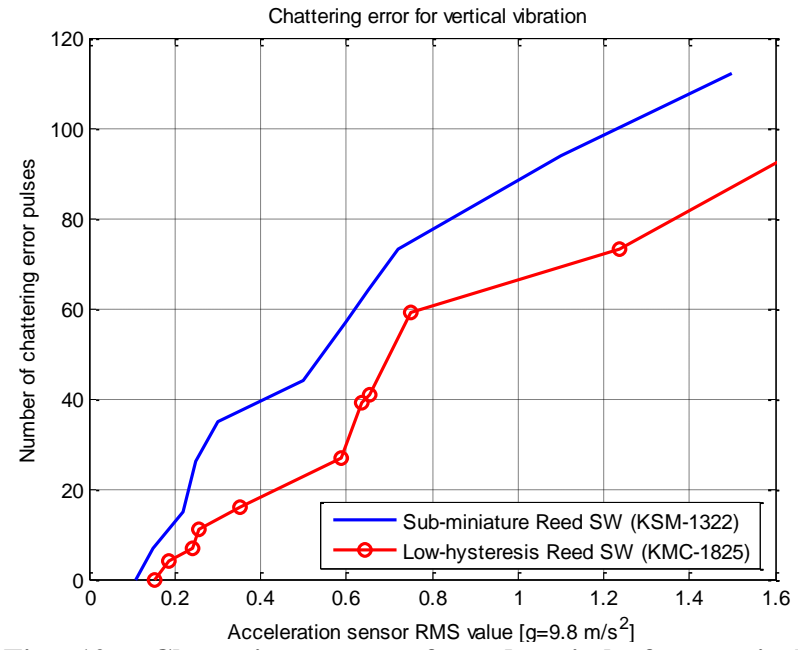

Fig. 12 Chattering error of reed switch for vertical vibration.

As shown in Figs. 10 and 12, X direction vibration is more sensitive (generating more chattering error pulses) than $\mathrm{Y}$ direction vibration because vibration direction axis is perpendicular to the reed springs of the reed switch. And $0.1117 \mathrm{~g}$ and $0.1528 \mathrm{~g}$ are the $\mathrm{X}$ and $\mathrm{Y}$ direction beginning RMS values of vibration for low-hysteresis reed switch (KMC-1825) respectively. As shown in the graphics, low-hysteresis reed switch was more stable against the external vibrations than sub-miniature reed switch.

\section{Conclusions}

The chattering error of reed switch sensor for AMR system was analyzed according to various types of external vibrations and shocks. Two kinds of reed switches were analyzed for vibration experiment. Low hysteresis reed switch was more stable than standard type reed switch against vibrations. As shown in an experiment result, $\mathrm{X}$ axis direction vibration is more sensitive (generating more chattering error pulses) than $\mathrm{Y}$ axis direction vibration. In further work, we have to design better performing reed switch against the vibrations such as designing 2 or 3 reed switches mounted on the mechanical type water flow meter. Also other approach is to change inside spring leaf structure of reed switch.

\section{Acknowledgment}

This research was financially supported by the Project No. 201501262003 "Sensitive and Errorless Electrical Security Fence by Using Minimal Friction Electricity in Coaxial Cable" of the National Research Foundation of Korea and by the Project No. COR_01/2015 "High Sensitive and Errorless Security Fence System" of the Mongolian Foundation for Science and Technology and by the Project No. 201304221024 "Security Fence Detection Accuracy of Cable Friction Electricity Sensor System" of the University of Seoul.

\section{References}

[1] Ayurzana, O., Kim, H., Park, Y., and Kwon, J. 2007. "Ubiquitous Telemetring System for Water Flow Meters Using Zigbee Module and CDMA Modem." ICUCT, January, 28-31, Aomori, Japan.

[2] Janh, W., and Gunther, W. 1995. Contact Materials for Reed Switches.

[3] Kobayashi, T., Hinohara, K., and Kawakita, C. 1991. 
"Magnetic Mechanical Design of Ultraminiature Reed Switches." IEEE 1991.

[4] Hinohara, K., Kobayashi, T., and Kawakita, C. 1993. "Influence of Contact Surface Conditions on Discharge Phenomena of Vacuum Reed Switches." IEEE Trans.
Component, Hybrids and Manufacturing Technology 16 (2).

[5] Kachroo, P., and Tomizuka, M. 1996. "Chattering Reduction and Error Convergence in the Sliding-Mode Control of a Class of Nonlinear Systems." IEEE Trans. on Automatic Control 41 (7). 